\title{
Candida endophthalmitis and infection of costal cartilages
}

\author{
PATRICK C. HO AND DENIS M. O'DAY \\ From the Department of Ophthalmology, Vanderbilt University School of Medicine, Nashville, USA
}

SUMMARY We report a case of endogenous candida endophthalmitis in which the clinical diagnosis was supported by recovery of the organism from a costochondral abscess. The patient recovered on systemic antifungal therapy, and vitreous surgery was not required.

Endogenous candida endophthalmitis is being encountered with increasing frequency as an opportunistic infection. ${ }^{1}$ We describe herein a case in which the clinical diagnosis was supported by isolation of the yeast from culture of a costochondral abscess. The risks and complications of vitreous surgery were thereby obviated and the infection responded well to medical management.

\section{Case report}

A 51-year-old man with a diagnosis of systemic lupus erythematosus developed floaters and blurred vision in his right eye. His ophthalmologist, noting the presence of vitreous debris in the right fundus, administered a retrobulbar steroid injection and prescribed $1 \%$ prednisolone acetate drops every 2 hours. However, vision continued to deteriorate and he was admitted to Vanderbilt University Hospital. He was on chronic prednisolone therapy by mouth and had been treated intermittently with amphotericin and Mycostatin (nystatin) for oral candidiasis. A previous episode of staphylococcal infection in his left knee had responded to a prolonged course of methicillin 3 months earlier. There was no history of intravenous drug abuse.

On admission the best corrected visual acuity was $6 / 18$ right eye and 6/6 left eye. Biomicroscopy revealed a trace flare in the right anterior chamber. The vitreous was moderately infiltrated with inflammatory cells, and focal inflammatory lesions were evident along the superotemporal retinal vessel at the posterior pole. These lesions had the appearance of elevated cotton-white fluffy mounds. Smaller glistening yellow-white lesions were scattered along

Correspondence to Dr Denis M. O'Day, Department of Ophthalmology, Vanderbilt University Medical Center, Nashville, Tennessee 37232, USA. the inferotemporal vessel in the right eye and near the optic disc in the left eye. Numerous greyishwhite precipitates were also present on the detached posterior hyaloid face in the right eye. The patient was cushingoid, afebrile, and had a painless effusion of his left knee.

Multiple bacterial and fungal cultures of blood, urine, bone marrow aspirate, and left knee aspirate were negative. A diagnostic vitrectomy was therefore planned. However, further examination revealed a tender hard swelling in the right fourth costal cartilage adjacent to the sternum, $1 \mathrm{~cm}$ in diameter. Although a lesion could not be demonstrated radiographically the costal cartilage was excised on the third hospital day. An abscess was present and Gram stain of the pus showed numerous pseudohyphae and chlamydospores (Fig. 1). Candida albicans was subsequently recovered on culture. Treatment with intravenous amphotericin in gradually increasing dosage and 5-flucytosine $(150 \mathrm{mg} / \mathrm{kg} /$ day) by mouth was begun immediately. Therapy with corticosteroid was progressively reduced. The patient improved remarkably despite the development of pancytopenia and gastrointestinal haemorrhage, which responded well to intermittent cessation of the antifungal agents and transfusion of blood products. The focal retinal lesions gradually resolved, with progressive clearing of the vitreous. The patient was discharged 6 weeks after admission having received a total of $1.017 \mathrm{~g}$ amphotericin. Five months later his visual acuity was $6 / 9$ in both eyes.

\section{Discussion}

Haematogenous spread to the choroid and retina is generally recognised as the mechanism of dissemination in endogenous fungal endophthalmitis. ${ }^{1-3}$ However, blood and urine cultures are often negative, and the only clue to the diagnosis of 
Fig. 1 Pseudohyphae seen on Gram stain of purulent material from costochondral abscess.

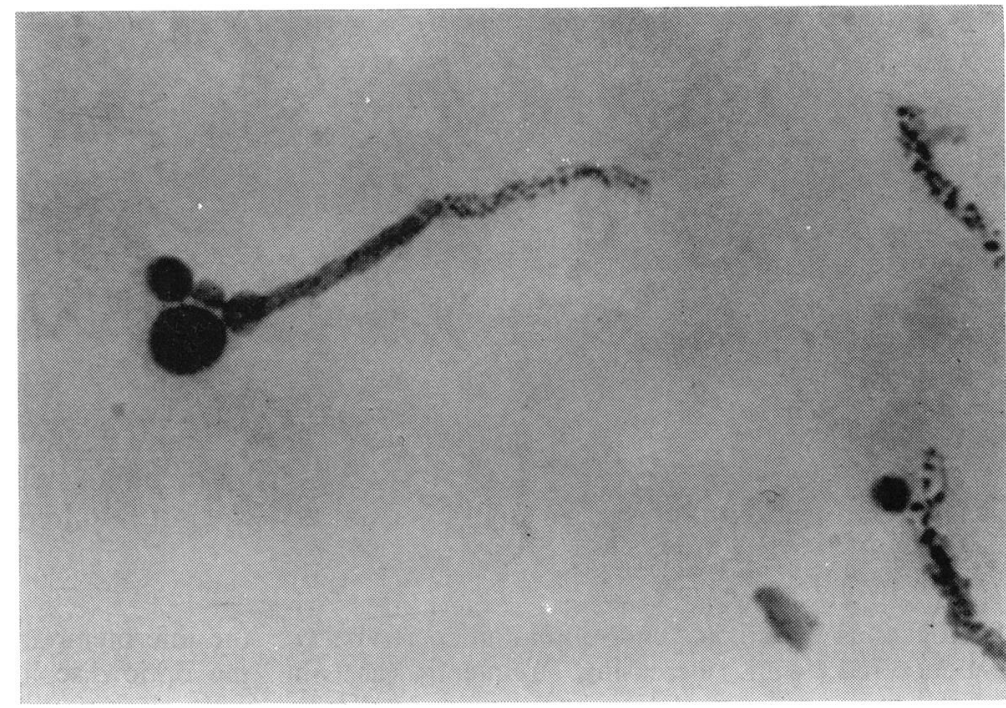

candidaemia may rest on the clinical appearance of fundal lesions. ${ }^{24}$

The high incidence of deep organ involvement in patients with metastatic candida endophthalmitis has been emphasised in a number of studies, with infection of such vital structures as liver, brain, meninges, kidney, and heart occurring in up to $80 \%$ of patients. ${ }^{13}$ In a recent report 2 cases of sternal osteomyelitis with adjacent costochondritis resulting from haematogenous dissemination of Candida albicans were documented. ${ }^{5}$ These lesions, as noted in our patient, may present as small seemingly innocuous masses, tender only to palpation. Unless a rigorous physical examination is made they may easily be overlooked.

Closed vitreous surgery is being increasingly advocated in the management of microbial endophthalmitis. Despite a steady advance in technical sophistication serious complications continue to occur. ${ }^{6}{ }^{7}$ It is not known whether the inflamed eye is more at risk of these complications, but in view of the potential for excellent recovery from candida endophthalmitis with noninvasive techniques ${ }^{8} 9$ it would seem wise to avoid surgical manipulation whenever possible. A careful search for infected sites elsewhere in the body more amenable to biopsy may render diagnostic vitrectomy unnecessary. Therapeutic vitrectomy can then be reserved for those cases where there is progression of the intra- ocular inflammation despite systemic and periocular antifungal therapy.

This work was supported in part by NIH grant EY 01621 and an unrestricted grant from Research to Prevent Blindness, Inc.

\section{References}

1 Edwards JE, Foos RY, Montgomerie JZ, Guze LB. Ocular manifestations of candida septicemia. Review of 76 cases of hematogenous candida endophthalmitis. Medicine 1974; 53: 47-75.

2 Robertson DM, Riley FC, Hermans PE. Endogenous candida oculomycosis. Arch Ophthalmol 1974; 91: 33-8.

3 Griffin JR, Pettit TH, Fishman LS. Blood-borne candida endophthalmitis. Arch Ophthalmol 1973; 89: 450-6.

4 Stone RD, Irvine AR, O'Connor GR. Candida endophthalmitis. Ann Ophthalmol 1975; 7: 757-62.

5 Thomas FE, Martin CE, Fisher RD. Candida albicans infection of sternum and costal cartilages. Ann Thorac Surg 1977; 23: 163-6.

6 Faulborn T, Conway BP, Machemer R. Surgical complications of pars plana vitreous surgery. Ophthalmology 1978; 85: 116-26.

7 Ghartey KN, Tolentino FI, Freeman HM, McMeel JW, Schepens CL, Aiello LM. Closed vitreous surgery XVII. Results and complications of pars plana vitrectomy. Arch Ophthalmol 1980; 98: 1248-52.

8 Elliott JH, O'Day DM, Gutow GS, Podgorski SF, Akrabawi PA. Mycotic endophthalmitis in drug abusers. Am J Ophthalmol 1979; 88: 66-72.

9 Blumenkranz MS, Stevens DA. Therapy of endogenous fungal endophthalmitis. Arch Ophthalmol 1980; 98: 1216-20. 